\title{
RADIOGRAPHIC EVALUATION OF COVID 19 PNEUMONIA- A RETROSPECTIVE OBSERVATIONAL STUDY IN A DEDICATED COVID 19 HOSPITAL IN CENTRAL INDIA.
}

\begin{tabular}{ll} 
Dr.Aarti Anand & $\begin{array}{l}\text { Hod And Prof, Dept Of Radiodiagnosis, GMCH Nagpur, Maharashtra, } \\
\text { India. }\end{array}$ \\
\hline Dr. Sakshi Kalra & $\begin{array}{l}\text { Junior Resident Doctor, Dept Of Radiodiagnosis, GMCH Nagpur, } \\
\text { Maharashtra, India. }\end{array}$ \\
\hline Dr. Srijit Saha* & $\begin{array}{l}\text { Junior Resident Doctor, Dept Of Radiodiagnosis, GMCH Nagpur, } \\
\text { Maharashtra, India. }{ }^{*} \text { Corresponding Author }\end{array}$ \\
\hline \hline
\end{tabular}

\section{ABSTRACT Context: Role of chest xray to aid in the diagnosis and prognosis of COVID 19 pneumonia.}

Aims: To evaluate the imaging features of routine chest X-ray in patients referred for novel Coronavirus

2019 infection.

Settings And Design: Retrospective observational study in a dedicated COVID 19 hospital in Central India

Methods And Materials: RT-PCR positive patients for SARS-CoV-2 infection were evaluated. Demographic and clinical data were recorded. All the CXR images were evaluated for the following findings: reticular opacities, alveolar opacities (AO) alone or in combination, consolidation and/or pleural effusion. We stratified patients in groups according to the time interval between day of admission and X-ray imaging and according to clinical presentation.

Statistical Analysis: Continuous variableswereexpressedasmean \pm SDvalues.Thefrequency of the radiographic findings was expressed as the number of occurrences and percentage in every single cluster, compared using chi square test.

Results: A total of 486 patients were tested positive for SARS-CoV-2. Lung lesions primarily manifested in $40 \%$ of the patients out of which the most frequent alteration wasconsolidations (41\%) followed by $A O(28 \%)$, pleural effusion( $18.5 \%$ ) and reticular opacities (11\%) and more frequently bilateral (59\%)with a peripheral predominance (83\%).Among paediatric patients, $20 \%$ CXR showed positive findings, out of which the most frequent alteration was $A O(54 \%)$.For the patients whose CXR was done within 3 days of admission more frequently had $\mathrm{AO}$ and reticular opacities, in comparison to those whose $\mathrm{x}$ ray was done after 3 days of admission.

Conclusions: The most common X-ray pattern is multifocal and peripheral, associated with consolidatory changes. Chest Xraycan be considered a reliable diagnostic tool especially in developingcountries with meagre resources.

\section{KEYWORDS : COVID-19, CXR, consolidations, AO}

\section{INTRODUCTION:}

As of August 18th, 2020, more than 21.8 million confirmed SARS-CoV-2 cases have been reported globally[1]. Of these, more than two million were from India.

Several early radiological studies analysed and described the chest computed tomography (CT) findings at the presentation and at different times throughout the disease course [2,3]. During the subsequent outbreak in the Western world, chest X-ray (CXR), together with arterial blood gas analysis and clinical presentation, in patients positive to RTPCR, was recommended as a useful and easily available tool to support the initial diagnosis and for the subsequent management of COVID-19 patients $[4,5]$. Nonetheless, data specifically addressing CXR findings in COVID-19 are still limited [6-11].

Our study aimed to evaluate the percentage of abnormal chest radiographs at different time intervals from the day of admission in COVID wards and to identify the type and distribution of radiographic alterations, the correlation between these radiologic alterations with $\mathrm{Sp02}$ and clinical presentation and their frequency at different times throughout the disease course of COVID-19 pneumonia.

\section{MATERIALS AND METHODS:}

2.1 Equipment Used:

All X-ray examinations were acquired as computed or digital radiographs using Allengers Mars 4.2following usual local protocols- with values of $\mathrm{mAS}-8$ and KV-62which are modified to $\mathrm{mAS} 15$ and KV 65 for patients with large body habitus. A standard chest X-ray was performed in anteroposterior projection or posteroanterior projection for patients who were able to stand, obtained in patients at the bedside, using portable X-ray units in the isolation wards.

\subsection{Selection And Description Of Participants}

Patients with a clinical suspicion of SARS-CoV-2 infection, based on referring physician's judgment, underwent the RTPCR test.Patients without clinical suspicion of SARS-CoV-2 infection were managed as appropriate.

Patients were eligible for study inclusion if they

1) underwent RTPCR test for SARS-CoV-2 on nasop haryngeal swab or oropharyngeal swabsand the results came out to be positive.

2) had at least one bedside chest X-ray as and when requisition received from the clinicians.

For each patient, the following demographic, clinical and imaging data were recorded:

a) age, b) sex, c) fever, d) cough, e) dyspneaf) onset of symptoms-Asymptomatic/Symptomatic, g) SpO2 values.

Patients were excluded out of the study if

1.) RT-PCR results came out to be negative .

2.) Technical errors in bedside CXR images.

3.) Previous history of radiographically significant lung disease.

4.) Antenatal patients.

\subsection{Subgrouping Of Patients:}

Patients were assigned into three groups, classified according to disease severity[12]: asymptomatic/mild, moderate, or severe.

These categories were defined as

a. "asymptomatic" if they had no symptoms of disease

b. "mild" if they had mild clinical symptoms, but normal mean oxygen saturation in resting state.

c. "moderate" if they hadclinical symptoms (fever, cough, diarrhoea, etc.), andmean oxygen saturation in resting 
state $90-94 \%$

d. "severe" if they had one of the following spectrum of conditions: severe pneumonia/ARDS/sepsis/septic shock with mean oxygen saturation in resting state $\leq 90 \%$

The patients were then clustered into three groups based on the number of days between day of admission and the chest radiography: group $A$ (patients with chest radiographs acquired 0-3 days from the day of admission), group B (patients with chest radiographs acquired 3-6 days from the day of admission), group $C$ (patients with chest radiographs acquired $\geq 7$ days from the day of admission.

\subsection{Image Evaluation:}

A senior attending radiologist (with 20 years of experience in chest imaging) reviewed the chest radiographs in picture archiving and communication systems (PACS).

$\mathrm{X}$-ray images were assessed for the presence and distribution of parenchymal abnormalities including

1) alveolar opacities ( $\mathrm{AO}$ )which were defined as a hazy increase in lung attenuation with no obscuration of the underlying vessels

2) reticularopacities $(\mathrm{RO})$

3) consolidations which was defined as an area of opacification obscuring the underlying vessels.

4) any combination of the above three.

5) pleural effusions.

Within each hemithorax, the craniocaudal distribution of the lesions was evaluated on the basis of the involvement of the upper, middle, and lower fields. The middle field was defined as the lung area delimited by (included between) two horizontal lines at the level of the superior and inferior hilar horns, respectively; the superior field was defined as the lung area included between the horizontal line at the level of the upper hilar horn and the apical pleura; the lower field was defined as the lung area included between the horizontal line at the level of the inferior hilar horn and the diaphragm.

The horizontal distribution of the lesions was evaluated on the basis of the involvement of the peripheral zone only, the central zone only, or both. Central zones were defined as the central area within $2 \mathrm{~cm}$ from the lobar bronchial structures as far as visible; peripheral zones were defined as the remaining lung area between the central zones and the pleura.

Moreover, the lesions were defined as isolated, when focal lesions involved only one zone, multiple when multiple zones are involved, unilateral or bilateral.

\subsection{Ethics:}

The study plan was discussed with the ethical committee of our institution and as it is retrospective observational study, the ethical approval was waived off.

\subsection{Statistical Analysis:}

Continuous variables were expressed as mean \pm SD values. The frequency of the radiographic findings was expressed as the number of occurrences and percentage in every single cluster. p values $<0.05$ were considered significant.

\section{RESULTS:}

Demographic, clinical and laboratory data of the entire cohort A total of 486patients diagnosed with COVID-19 wereinitial lyrecruited to this study. Seven patients were excluded from the study because of images with poor quality. The remaining 479 patients from our hospital were assigned to three severitygroups (asymptomatic/mild, $\mathrm{n}=290$; moderate, $\mathrm{n}$ $=129$; severe/criticallyill, $\mathrm{n}=60$ ) and three groups based on the day of chest $\mathrm{x}$-ray from the day of admission as group $\mathrm{A}, \mathrm{B}$ and C.
The majority of patients were male $(283 ; 59.08 \%)$ with a mean age of 37 years $( \pm 36)$. Therewasmarkeddisparityinmedi anagebetween the three groups, manifesting as an increased age withincreased severity $(\mathrm{p}<0.001)$.

Fever (up to $80 \%$ ) and cough/ generalised weakness(up to $61 \%$ ) were the most common symptoms in symptomatic patients. Most of them accessed the emergency department within two days after the onset of clinical manifestati ons.[Table 1]

Table 1: Patient Demography And Symptomatology

\begin{tabular}{|c|c|c|c|}
\hline$\overline{\mathrm{AGE}}$ & $<15$ YEARS & 15-60 YEARS & $>60$ YEARS \\
\hline $\begin{array}{l}\text { NUMBER OF } \\
\text { PATIENTS }\end{array}$ & 67 & 328 & 84 \\
\hline MALE & $38(56.7 \%)$ & $195(59.4 \%)$ & $50(59 \%)$ \\
\hline $\begin{array}{l}\text { CLINICAL } \\
\text { SYMPTOMS }\end{array}$ & & & \\
\hline Asymptomatic & $60(89 \%)$ & $200(60.9 \%)$ & $15(17.8 \%)$ \\
\hline Fever & $2(3 \%)$ & 128(39\%) & $40(47.6 \%)$ \\
\hline Cough & $3(4.5 \%)$ & $60(18.2 \%)$ & $18(21.4 \%)$ \\
\hline $\begin{array}{l}\text { Generalized } \\
\text { weakness }\end{array}$ & - & $38(11.5 \%)$ & $4(4.7 \%)$ \\
\hline $\begin{array}{l}\text { Breathing } \\
\text { difficulty }\end{array}$ & $1(1.5 \%)$ & $40(12.2 \%)$ & $38(45.2 \%)$ \\
\hline Nasal congestion & $1(1.5 \%)$ & $24(7.3 \%)$ & $16(19 \%)$ \\
\hline Others & - & $10(3.1 \%)$ & $19(22.6 \%)$ \\
\hline
\end{tabular}

Analyses of laboratory test results for SpO2showed downward trends from asymptomatic/mild to severe/critically ill. Patients with severe disease tended to have more adverse events including admission to ICU, invasive mechanical ventilation ( $11 \%)$.

Among the total of 479 patients, 252 (52.6\%) had at least one alteration in one lung field. In $227 / 479$ patients (47.4\%), CXR was normal without any lesion. CXR showed at least one alteration in at least one lung field in $75 / 206$ patients $(36.4 \%)$ in group $A$, in $90 / 172$ patients $(52.3 \%$ ) in group $B$, in $87 / 101$ patients $(86.1 \%)$ in group $C$. The negative rate of chest radiographs was $131 / 206$ patients $(63.6 \%)$ in group $A, 82 / 172$ $(47.7 \%)$ in group $B, 14 / 101$ (13.9\%) in group C.

Alterations were bilateral in 150/252 patients (59.5\%) and unilateral in 102/252 (40.5\%) (52 in the right lung and 50 in the left lung). $\mathrm{AO}$, alone or in combination with other alterations, was present in $107 / 252$ patients (42.5\%); reticular alteration, alone or in combination with other findings, was present in $55 / 252$ patients (21.8\%); consolidation, alone or in combination with other findings, was present in $91 / 252$ patients $(36.1 \%)$, pleural effusion was present in $33 / 252$ patients( $13.1 \%)$. AO was significantly more frequent than consolidation and reticular alteration.

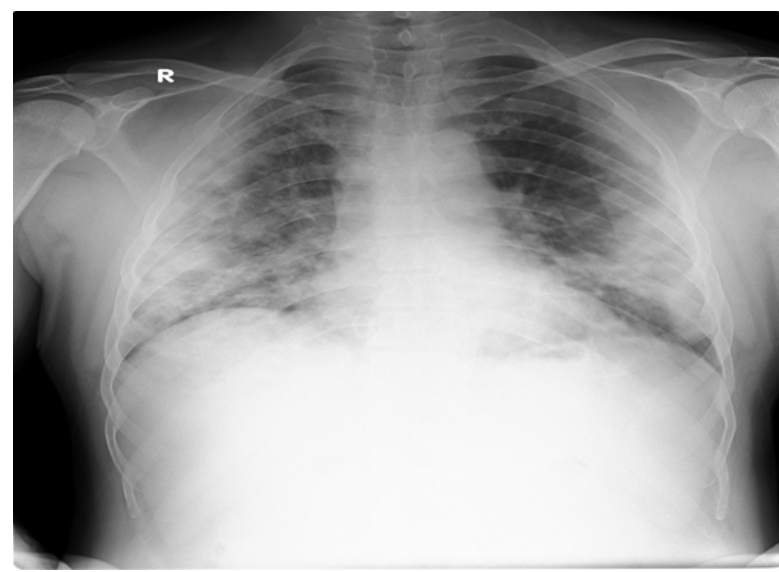

Figure 1: Chest Xray Showing Alveolar Opacities 
A significantly higher frequency of involvement of the lower fields compared to the middle fields and of the lower and middle fieldscompared to the upper fields was observed ( $p$ $<0.01$ in all cases). The exclusive involvement of the central zones was significantly less frequent than the exclusive involvement of the peripheral zones which was less than the involvement of both peripheral and central zones $(p<0.01$ in both cases)

In our study, in the patients having $\mathrm{SpO} 2$ values greater than $94 \%$ the radiographs consistently showed no abnormality or presence of $A O / R O$ in a single zone in 290/479 patients(60.5 $\%)$ whilein patients with $\mathrm{SpO} 2$ levels between $90-94 \%$, the radiographs showed patchy $\mathrm{AO}$ or consolidations in single or multizonal distribution in $129 / 479(27 \%)$ and in patients with SPO2 levels $<90 \%$, there was diffuse multilobar peripheral basal involvement of bilateral lungs showing confluent consolidations.[Illustration 1]

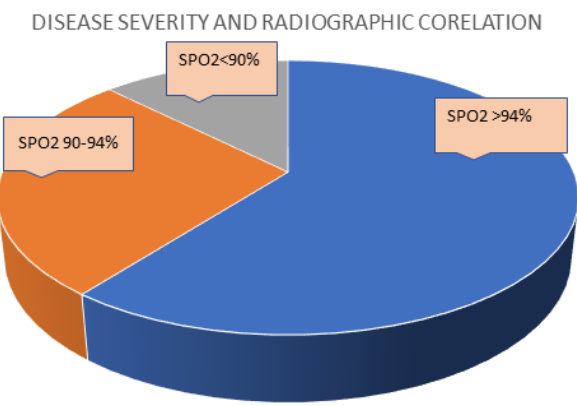

- no abnormality or presence of $\mathrm{AO} / \mathrm{RO}$ in a single zone

- patchy AO or consolidations in single or multizonal distribution

- diffuse multiobar peripher al basal involvement of bilate al lungs showing confluent consolidations

Illustration 1: Subgrouping On The Basis Of Clinical Severity

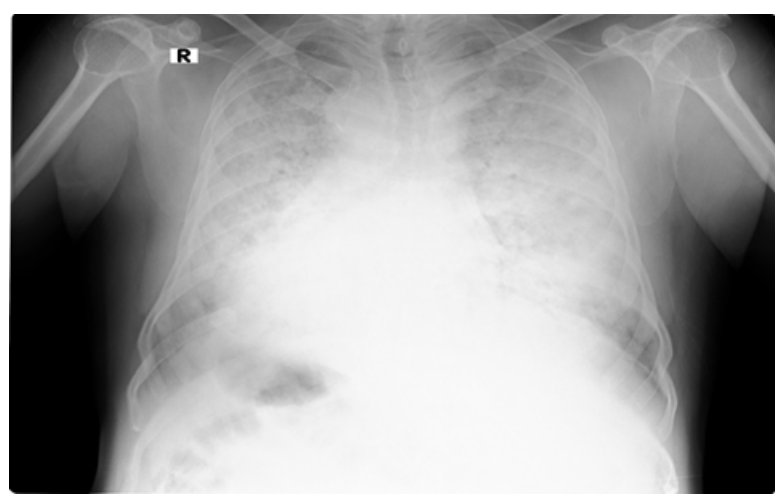

Figure 2: Chest Xray Showing Bilateral Confluent Consolidations

Chest Radiography Evaluation Of Subgroups:

Bilateral alterations were present in $26 / 75$ patients $(34.6 \%$ ) in group $A$, in $55 / 90$ patients $(61.1 \%$ ) in group $B$, in $69 / 87$ patients $(79.3 \%)$ in group $C$; when alterations were unilateral, no significant difference was observed between the left and right lungs. $\mathrm{AO}$, alone or in combination with other alterations, was presentin30/75 patients(40\%)ingroupA,in35/90 patients (38.9\%)ingroupB,in42/87patients(48.2\%)ingroupC. Reticular alteration, alone or in combination with other alterations, was present in $28 / 75$ patients $(37.3 \%$ ) in group $\bar{A}$, in $17 / 90$ patients (18.9\%) in group $B$, in $10 / 87$ patients (11.5\%)ingroupC.

Consolidation, alone or in combination with other alterations, was present in $9 / 75$ patients $(12 \%)$ in group $A$, in $22 / 90$ patients ( $24.4 \%$ ) in group $B$, in $60 / 87$ patients $(68.9 \%)$ in group $C$. [Table 2]
Table 2: Radiographic Alterations

\begin{tabular}{|l|l|l|l|}
\hline & Group $\mathbf{A}$ & Group B & Group C \\
\hline TOTAL ,n (\%) & $206(43 \%)$ & $172(35.9 \%)$ & $101(21.1 \%)$ \\
\hline NEGATIVE CXR & $131(63.6 \%)$ & $82(47.7 \%)$ & $14(13.9 \%)$ \\
\hline POSITIVE CXR & $75(36.4 \%)$ & $90(52.3 \%)$ & $87(86.1 \%)$ \\
\hline $\begin{array}{l}\text { ALVEOLAR } \\
\text { OPACITIES(AO) }\end{array}$ & $30(40 \%)$ & $32(35.6 \%)$ & $23(26.4 \%)$ \\
\hline $\begin{array}{l}\text { RETICULAR } \\
\text { OPACITIES(RO) }\end{array}$ & $28(37.3 \%)$ & $15(16.7 \%)$ & - \\
\hline CONSOLIDATION & $9(12 \%)$ & $17(18.9 \%)$ & $31(35.6 \%)$ \\
\hline AO+ CONSOLIDATION & - & $3(3.3 \%)$ & $19(21.8 \%)$ \\
\hline RO+ CONSOLIDATION & - & $2(2.2 \%)$ & $10(11.5 \%)$ \\
\hline PLEURAL EFFUSION & $8(10.7 \%)$ & $21(23.3 \%)$ & $4(4.6 \%)$ \\
\hline
\end{tabular}

In group $A$, lower lung zone involvement was noted in majority of the patients i.e. $61 / 75(81.3 \%)$. In group $B$, the dominant pattern wasboth lower and mid lung zoneinvolvement and was seen in55/90(61.1\%) patients. In group C, 68/87(78.1\%) patients showed involvement of the entire lung fields which was not seen in the other groups in such high proportions.

The exclusive involvement of the peripheral zones was observed in $50 / 75$ patients (66.7\%) in group $A$, in $63 / 90(70 \%)$ in group B, in 17/87 (19.5\%) in group C while 70/87(80.5\%) patients in group $\mathrm{C}$ showed involvement of both central and peripheral lung zones. [Illustration2 and 3]

HORIZONTAL DISTRIBUTION

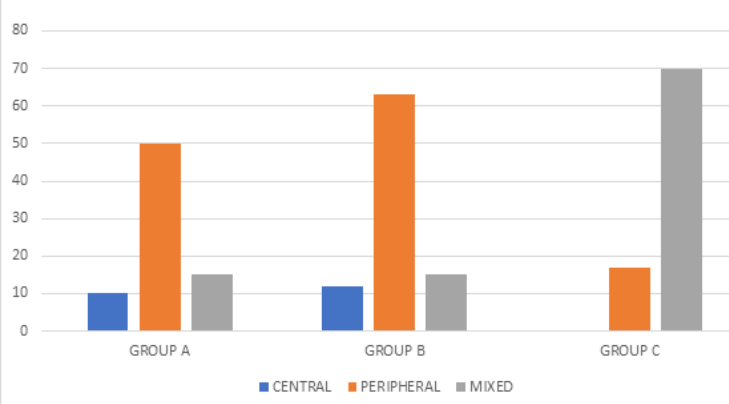

Illustration 2: Horizontal Distribution Of Radiographic Alterations In Different Groups

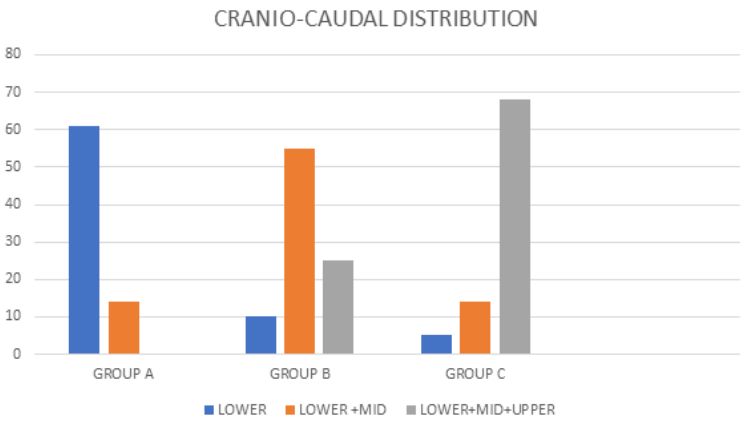

Illustration 3: Craniocaudal Distribution Of Radiographic Alterations In Different Groups

In our study, the age group of the patients varied from 5 months to 75 years; out of which 67 patients were below 15 years of age. Amongst these, the positive CXR rate was $13 / 67(19.4 \%)$. On further subgrouping, the positivity rate in group A was $7 / 45(15.5 \%)$, group B 6/20(30\%) and in group C $0 / 2(0 \%)$. AO were found in group $A$ in $5 / 7$ patients $(71.4 \%)$ and in group B 2/6 (33.3\%). Consolidatory changes were found in group $A$ in $1 / 7$ patients $(14.3 \%)$ and in group $B$ in $4 / 6$ patients(66.7\%). Pleural effusion was found in only 2 patients 
in group A.[Table 3]

Table 3: Radiographic Alterations In Patients < 15 Yrs Of Age

\begin{tabular}{|l|l|l|l|}
\hline & Group A & Group B & Group C \\
\hline TOTAL ,n (\%) & $45(67 \%)$ & $20(30 \%)$ & $2(3 \%)$ \\
\hline NEGATIVE CXR & $38(84.4 \%)$ & $14(70 \%)$ & $2(100 \%)$ \\
\hline POSITIVE CXR & $7(15.6 \%)$ & $6(30 \%)$ & - \\
\hline AO & $5(71.4 \%)$ & $2(33.3 \%)$ & - \\
\hline RETICULAR OPACITIES(RO) & - & - & - \\
\hline CONSOLIDATION & $1(14.3 \%)$ & $4(66.7 \%)$ & - \\
\hline PLEURAL EFFUSION & $2(28.6 \%)$ & - & - \\
\hline
\end{tabular}

\section{DISCUSSION:}

According to WHO guidelines, diagnostic testing for SARSCoV-2 is fundamental, in particular, to avoid transmission, track the epidemiology, and, finally, manage patients correctly. On the other hand, due to the large number of cases suspected for SARS-CoV-2 infection, laboratory detection is time-consuming and may not be quickly available for all people with suspected infection, with a time interval between 2 hours and 2 days. Therefore, we assumed that chest X-ray can achieve the potential role of a screening test as and when the need arises in countries like Indiawith high disease prevalence but limited resources.

By analysing international literature the proportion of patients with abnormal initial radiographic findings was 78.3-82.4\% in SARS [13], 83.6 \% in MERS [14], while only in $33 \%$ of cases in Korean SARS-CoV-2 related pneumonia [15], and in $60 \%$ of Chinese patients [16]. In our series, investigating Indian patients, the percentage of positive radiographs came to be $52.6 \%$.Wong et al [7] reviewed $255 \mathrm{CXR}$ in 64 patients, describing the time course of the radiographicfindings of COVID-19 pneumonia and reporting an overall rate of negative baseline CXR examinations of $31 \%$. However, in our experience, the overall rate of normal CXR Finding sin patients with positiveRT-PCRwas $47.4 \%$, possibly owing to the higher number of younger asymptomatic patients in India.

Bernheim et al[17]reported the percentage of normal chest $\mathrm{CT}$ in patients clustered on the base of the timing of symptom onset and observed a rapid decrease from $56 \%$ at $0-2$ days to $9 \%$ at $3-5$ days and $4 \%$ at $6-12$ days. Clustering our patients on the base of the timing of chest $x$ ray from the day ofadmission, the rate of normal CXR progressively decreased from $63.6 \%$ (0-3 days from the day of admission) to $47.7 \%$ (3-6 days), $13.9 \%$ ( $\geq 7$ days). Although the two cohorts are not directly comparable due to different selection criteria and different diagnostic modalities, we observed a decreasing trend in the rate of negative CXR through the different time intervals.

In our radiographic series, consistent with previous studies, SARS-CoV-2 was more often found in men than in women; however the difference was not statistically significant. In our study, the most frequent alterations wasAO, alone or in combination with other alterations, resembling the radiographic appearance described in other coronavirusrelated pneumonias [18-20]. Furthermore, the distribution of the lesions in the middle and lower fields and that of the relatives paring of the superior fields are similar to the pattern of distribution described in HINl influenza pneumonia [21]. Our results are not in line with the recent report of Wong et al. [7] because in our series too we found a higher number of $A O$ than consolidation and reticular opacities.

In the first 3 days from the day of admission, both $A O$ and reticular alterations weremorefrequent, while after this period, consolidation came to be predominant. Consolidation was less frequent than the other two alterations in the early phase of the disease. Our data suggest that early alterations are predominantly $A O$ and reticular opacities, whereas consolidations started to increase from the intermediate phase. As compared to the previous studies, our study had a fair percentage of patients with pleural effusion, possibly parapneumonic in etiology. Considering the distribution of the lesions on both the lungs and on the axial and craniocaudal plane, our results are in line with the data reported in CXR studies and in CT studies in the literature $[2,7,22,23]$. In accordance with the observations of Wong et al [7], in the majority of our patients, the alterations were bilateral(59.5\%), andin patients with unilateral lesions, no predominance was observed between left and right. The exclusive peripheral involvement and the combination of peripheraland central distributions were significantly more frequent than exclusive central distribution; with significant differences in the proportion of distribution according to the day of CXR from admission. Peripheral involvement was seen mostly in Group A and $B$ while group $C$ showed more or less a mixed pattern of involvement. Several studies reported a predominant, although not significant,localizationofthelesions inthelower lobes [17,24]; our results confirm this observation and measured a significantly higher frequency of involvement of the middle and lower zones compared to the upper zonessimilar to SARS and MERS imaging. Our patients had a lower mean age than those reported in the literature, reflecting demographic differences between India and the Western countries.

In our study, we observed that the distribution of radiographic alterations were seen to be having a correlation with the SPO2 levels, no such finding has yet been mentioned in the literature that we could find. Patients having $\mathrm{SpO} 2$ values greater than $94 \%$ consistently showed no abnormality or presence ofAO/ $\mathrm{RO}$ in a single zone while in patients with $\mathrm{SpO} 2$ levels between $90-94 \%$, the radiographs showed patchy AO or consolidations in single or multizonal distribution and in patients with SPO2 levels $<90 \%$, there was diffuse multilobar peripheral basal involvement of bilateral lungs showing confluent consolid ations.

In paediatric patients, the CXR positivity rate was significantly lower than the adult population which is in line with the milder clinical presentation in the younger population, most of whom acquired the infection from contact with their parents who were positive for COVID 19. Most of the paediatric patients were imaged in first 6 days of admission and the predominant finding was found to be AO followed by consolidation. Pleural effusion was found in 2 patients.

In fact, we found out that chest X-ray sensitivity decreased when symptoms appeared $\leq 6$ days before performing imaging technique with a value of only $43.6 \%$ while in case of a longer course of the disease the sensitivity increased up to $86 \%$.

Moreover the subdivision of SARS-CoV-2 patients according to age ( $\leq$ and $>60$ years) allows to establish the diagnostic performance changes: inyounger patientschestX-rayshowed a low sensitivity (47 \%) while sensitivity increased in older patients $(65 \%)$, maybe due to the presence of others comorbidities.

The purpose of this study was to analyse and describe the type, frequency, and distribution of CXR findings in COVID-19 pneumonia. Therefore, the main limitation of the study is the lack of data about the specificity of the CXR findings in COVID-19 towards its main differential diagnoses (other viral pneumonia, interstitial lung disease, cardiogenic pulmonary edema, acute lung injury), and the lack of correlation between CXR and CT findings. Our study focused on the CXR at the time of received requisition of CXR, and the subsequent examinations of each patient were not considered; hence, the radiographic differences between the groups do not reflect the 
course of the radiographic evolution over time, but rather the different radiographic presentations at different time intervals from the day of admission. Another limitation of the present study is due to the lower quality of bedside radiographs compared to the PA radiographs. A chest radiograph can establish the presence of pneumonia, define its extension and location, and can also diagnose complications like pleural effusion, while CT can show abnormalities that are not detectable with chest radiograph, especially the less extended, due to its higher sensitivity.It is well known that patients without infiltration observed on radiograph and with unsure diagnosis greatly benefited from CT scan, however, we weighed this consideration against the importance of urgent reporting.

Even if different studies evaluated the role of CT as a routine imaging modality for diagnosis or screening, the use of CT is rather complex, especially in the Emergency Department, first of all, due to time-consuming decontamination procedures to be completed in the CT room among different patients [22]. The Radiology Scientific Expert Panel suggests that, after C Timaging, the room down timeis typically between $30 \mathrm{~min}$ to $\mathrm{lh}$ for room decontamination [25], suggesting that chest X-ray may beconsidered to minimize the risk of cross-infection. Implications derived from our results are that the use of chest radiograph may help in determining both diagnosis and treatment, and deciding the best management in patients with suspected SARS-CoV-2related pneumonia in the emergency setting. Therefore, we believe that a strategy that promotes chest radiograph as imaging technique in targeted patients in the ED would decrease time to diagnosis and may even, sooner, reduce global radiation dose exposure by limiting particular CT examinations. In conclusion, our observation is that the X-ray manifestations of SARS-CoV-2 infection are quite typical, with an acceptable overall sensitivity of $52 \%$ for SARS-CoV-2-related pneumonia. Sensitivity can be even higher when symptoms had started more than 6 days before, at the expense of lesser specificity, and slightly higher in older patients in comparison to younger ones

\section{REFERENCES:}

1. World HealthOrganization (2020) WHODirector-General's opening remarks at the media briefing on COVID-19. World Health Organization, Geneva Available via https://www.who.int/dg/speeches/detail/who-director-generals-opening-remarks-at-the-media-briefing-on-covid-19---1 1-march-2020

2. Shi $\mathrm{H}, \mathrm{Han} X$, Jiang $N$, Cao $Y$, Alwalid $O, G u$ J et al. Radiological findings from 81 patients with COVID-19 pneumonia in Wuhan, China: a descriptive study. The Lancet Infectious Diseases. 2020;20(4):425-434

3. Ng M, Lee E, Yang J, Yang F, Li X, Wang H et al. Imaging Profile of the COVID19 Infection: Radiologic Findings and Literature Review. Radiology: Cardiothoracic Imaging. 2020;2(1):e200034.

4. Song F, Shi N, Shan F, Zhang Z, Shen J, Lu H et al. Emerging 2019 Novel Coronavirus (2019-nCoV) Pneumonia. Radiology. 2020;295(1):210-217.

5. Rubin G, Ryerson C, Haramati L, Sverzellati N, Kanne J, Raoof S et al. The Role of Chest Imaging in Patient Management during the COVID-19 Pandemic: A Multinational Consensus Statement from the Fleischner Society. Radiology. 2020;296(1):172-180.

6. Vancheri S, Savietto G, Ballati F, Maggi A, Canino C, Bortolotto C et al. Radiographic findings in 240 patients with COVID-19 pneumonia: timedependence after the onset of symptoms. European Radiology. 2020:

7. Wong $\mathrm{H}$, Lam $\mathrm{H}$, Fong $\mathrm{A}$, Leung $\mathrm{S}$, Chin $\mathrm{T}$ Lo $\mathrm{C}$ et al. Frequency and Distribution of Chest Radiographic Findings in COVID-19 Positive Patients. Radiology. 2019;::201160.

8. Salehi S, Abedi A, Balakrishnan S, Gholamrezanezhad A. Coronavirus Disease 2019 (COVID-19): A Systematic Review of Imaging Findings in 919 Patients. American Journal of Roentgenology. 2020;215(1):87-93.

9. Vancheri S, Savietto G, Ballati F, Maggi A Canino C, Bortolotto C et al Radiographic findings in 240 patients with COVID-19 pneumonia: timedependence after the onset of symptoms. European Radiology. 2020;

10. Wang Y, Luo H, Liu S, Huang S, Zhou Z, Yu Q et al. Dynamic evolution of COVID-19 on chest computed tomography: experience from Jiangsu Province of China. European Radiology. 2020;

11. Ippolito D, Maino C, Pecorelli A, Allegranza P, Cangiotti C, Capodaglio C et al. Chest X-ray features of SARS-CoV-2 in the emergency department: a multicenter experience from northern Italian hospitals. Respiratory Medicine. 2020;170:106036

12. https://www.mohfw.gov.in/pdf/RevisedNationalClinicalManagementGuid elineforCOVID1931032020.pdf

13. Wong K, Antonio G, Hui D, Lee N, Yuen E, Wu A et al. Severe Acute Respiratory Syndrome: Radiographic Appearances and Pattern of Progression in 138 Patients. Radiology. 2003;228(2):401-406.

14. Das K, Lee E, Langer R, Larsson S. Middle East Respiratory Syndrome Coronavirus: What Does a Radiologist Need to Know?. American Journal of
Roentgenology. 2016;206(6):1193-1201.

15. Yoon S, Lee K, Kim J, Lee Y, Ko H, Kim K et al. Chest Radiographic and CT Findings of the 2019 Novel Coronavirus Disease (COVID-19): Analysis of Nine Patients Treated in Korea. Korean Journal of Radiology. 2020;21(4):494.

16. https://www.who.int/emergencies/diseases/novel-coronavirus-2019/tec hnical-guidance

17. Bernheim A, Mei X, Huang M, Yang Y, Fayad Z, Zhang N et al. Chest CT Findings in Coronavirus Disease-19 (COVID-19): Relationship to Duration of Infection. Radiology. 2020;295(3):200463.

18. Müller N, Ooi G, Khong P, Nicolaou S. Severe Acute Respiratory Syndrome: Radiographic and CT Findings. American Journal of Roentgenology. 2003;181(1):3-8

19. Franquet T. Imaging of Pulmonary Viral Pneumonia. Radiology. 2011;260 (1):18-39.

20. Koo H, Lim S, Choe J, Choi S, Sung H, Do K. Radiographic and CT Features of Viral Pneumonia. RadioGraphics. 2018;38(3):719-739.

21. Aviram G, Bar-Shai A, Sosna J, Rogowski O, Rosen G, Weinstein I et al. HIN Influenza: Initial Chest Radiographic Findings in Helping Predict Patient Outcome. Radiology. 2010;255(1):252-259

22. Pan F, Ye T, Sun P, Gui S, Liang B, Li L et al. Time Course of Lung Changes at Chest CT during Recovery from Coronavirus Disease 2019 (COVID-19). Radiology. 2020;295(3):715-721.

23. Chung M, Bernheim A, Mei X, Zhang N, Huang M, Zeng X et al. CT Imaging Features of 2019 Novel Coronavirus (2019-nCoV). Radiology. 2020;295(1):202207.

24. Zu Z, Jiang M, Xu P, Chen W, Ni Q, Lu G et al. Coronavirus Disease 2019 (COVID-19): A Perspective from China. Radiology. 2020;:200490.

25. Mossa-Basha M, Meltzer C, Kim D, Tuite M, Kolli K, Tan B. Radiology Department Preparedness for COVID-19: Radiology Scientific Expert Panel. Radiology. $2020 ;: 200988$. 